\title{
Elicitation Enhanced the Production of Phenolic Compounds and Biological Activities in Hairy Root Cultures of Bitter melon (Momordica charantia L.)
}

\author{
Ill-Min Chung ${ }^{1}$, Muthu Thiruvengadam ${ }^{1}$, Kaliyaperumal Rekha ${ }^{1}$, Govindasamy \\ Rajakumar $^{1^{*}}$. \\ ${ }^{I}$ Konkuk University - Applied Bioscience, Korea (the Republic of).
}

\begin{abstract}
Momordica charantia (Cucurbitaceae) is an important vegetable and also medicinal crop which produces the bioactive compounds for various biological activities with potential uses in human health. The present investigation relates to elicitors of jasmonic acid (JA) and salicylic acid (SA) to enhance biomass accumulation and phenolic compound production in hairy root cultures of M. charantia. Hairy root cultures were elicited with JA and SA at 0 , 25, 50 and $100 \mu M$ concentrations respectively. The adding of elicitation to the hairy root cultures on the 15th day of culture and the roots were harvested on day 25. Cultures supplemented with $100 \mu \mathrm{M} J A$ and SA enhanced the phenolic compounds significantly compared to that of non-elicited hairy root cultures. The biomass of hairy root culture significantly increased by SA whereas decreased in JA elicitation at $100 \mu M$. JA and SA-elicited hairy root cultures significantly produced a higher amount of phenolic compounds (12811.23 and $11939.37 \mu \mathrm{g} / \mathrm{g})$, total phenolic (4.1 and $3.7 \mathrm{mg} / \mathrm{g})$ and flavonoid $(3.5$ and $3.2 \mathrm{mg} / \mathrm{g}$ ) contents than non-elicited hairy root cultures (10964.25 $\mu \mathrm{g} / \mathrm{g}, 2.8$ and $2.5 \mathrm{mg} / \mathrm{g}$ ). JA and SA-elicited hairy root cultures were significantly higher antioxidant activity of DPPH (84 and 78\%), reducing potential (0.53 and 0.48), phosphomolybdenum (3.6 and $3.2 \mathrm{mg} / \mathrm{g}$ ) and ferrous ion chelating assays (80 and 74\%) than non-elicited hairy root cultures. The higher antimicrobial and anticancer activity were exhibited in JA and SA-elicited than non-elicited hairy root cultures. This protocol can be developed for the production of phenolic compounds from JA and SA-elicited hairy root cultures.
\end{abstract}

Key words: Anticancer activity, Antioxidant activity, Antimicrobial activity, Elicitation, Hairy root cultures, Phenolic compounds

\footnotetext{
*Author for correspondence: microlabsraj@gmail.com
} 


\section{INTRODUCTION}

Bitter melon (Momordica charantia L.) is a climber belonging to the family Cucurbitaceae and widely distributed throughout tropical and subtropical regions on all continents. It is cultivated in tropical areas of Asia, Amazon, Africa, Caribbean, North, Central and South America for consumed as a vegetable as well as medicine. Bitter melon has been used traditionally as medicine in developing countries like Brazil, China, Colombia, Cuba, Ghana, Haiti, India Mexico, Malaya, New Zealand, Nicaragua, Panama and Peru. Several studies have revealed that bitter melon is used for numerous beneficial effects on health such as anticancer, antiviral, antimicrobial, anti-inflammatory, analgesic, hypolipidemic and hypocholesterolemic effects ${ }^{2}$. However, the plant has received increased attention due to the presence of significant amounts of pharmacologically important bioactive compounds. Phenolic compounds are considered as essential and universal compounds in the plant kingdom. They are derived from central or primary metabolic processes in plants ${ }^{3}$. Phenolic compounds, such as flavonols, hydroxybenzoic and hydroxycinnamic acids, that have been identified in bitter melon showed better antioxidant activity and pharmacological functions ${ }^{4}$. Plant cell, organ, and hairy root cultures are considered as an alternative for the supply of bioactive pharmaceutical secondary metabolites ${ }^{5}$. Various systems have been demonstrated for the synthesis and production of pharmaceutically important bioactive compounds. However, among these systems, Agrobacteriummediated gene transfer has been widely studied as a strategy for producing hairy root lines with high yield for bioactive compounds ${ }^{6}$. Genetically transformed hairy roots established by using the transformation of a plant cell with A. rhizogenes offer a promising system for secondary metabolite production due to their inherent genetic and biochemical stability ${ }^{7}$. Yield enrichment approach involving the exogenous addition of elicitor molecules is one of the most promising ways employed to enhance the productivity of phytochemicals in in vitro cultures ${ }^{8}$. Elicitation is widely employed as an approach to simulate the stress effects which could lead to a physiological response involving the synthesis and accumulation of secondary metabolites in the transformed hairy roots ${ }^{9}$. Previously it has been reported that elicitation of hairy root cultures and improvement in biosynthetic competence in vitro were reported $^{10,11,12}$. The involvement of natural phytohormones, such as jasmonic acid (JA) and salicylic acid (SA) in signal transduction cascades has led to the use of these molecules as exogenous inducers of defense mechanisms for boosting accumulation of various secondary metabolites in some plant in vitro systems ${ }^{7}$. Several findings on the production of many variable secondary metabolites using JA and SA elicitors in hairy root cultures of various medicinal plants ${ }^{13,14,15}$. Our earlier study found that the hairy root line induced by $A$. rhizogenes strain KCTC 2703 on the leaf explants of $M$. charantia, were able to produce the higher amount of phenolic compounds than the non-transformed roots ${ }^{16}$. Therefore, elicitation will be further described in the present study for improved production of phenolic compounds in these transformed hairy roots. The present communication reports for the first time the influence of elicitors (JA and SA) on biomass and enhanced phenolic compounds (flavonols, hydroxybenzoic and hydroxycinnamic acids derivatives) production from hairy root cultures of bitter melon. We also evaluated the determination of total phenolic and flavonoid contents and biological activities (antioxidant, antimicrobial and anticancer) from elicited (JA and SA) and nonelicited hairy root cultures of bitter melon.

\section{MATERIALS AND METHODS}

\section{Plant material and establishment of hairy root culture}

Seeds of bitter melon were received from Guangzhou Danong Horticultural Seed Co., Ltd., Korea. Agrobacterium rhizogenes strain KCTC 2703 was used for hairy root induction. The hairy root cultures of $M$. charantia was established and molecularly confirmed by our previous report ${ }^{16}$. Hairy roots $(500 \mathrm{mg})$ were in $250 \mathrm{~mL}$ Erlenmeyer's flasks containing $50 \mathrm{~mL}$ of $\mathrm{MS}$ medium $^{17}$ supplemented with $30 \mathrm{~g} / \mathrm{L}$ sucrose. The cultures were kept under continuous agitation at $100 \mathrm{rpm}$ in an orbital shaker and incubated at $25 \pm$ $2{ }^{\circ} \mathrm{C}$ with a $16 \mathrm{~h}$ photoperiod $\left(40 \mu \mathrm{mol} \mathrm{m} \mathrm{m}^{-2} \mathrm{~s}^{-1}\right)$ provided by $40 \mathrm{~W}$ white fluorescent lamps. The roots were subcultured every two weeks.

Elicitation of jasmonic acid (JA) and salicylic acid (SA) in hairy root cultures 
Different concentrations of JA and SA $(0,25,50$ and $100 \mu \mathrm{M}$ ) were added to the culture medium on day 15 of culture. Non-elicited cultures were considered as control. The elicitors were diluted with ethanol and then filter-sterilized. The same amount of ethanol was added to the control culture. All the cultures were kept under continuous agitation at $100 \mathrm{rpm}$ in an orbital shaker and incubated at $25 \pm 2{ }^{\circ} \mathrm{C}$, with a $16 \mathrm{~h}$ photoperiod $\left(40 \mu \mathrm{mol} \mathrm{m} \mathrm{m}^{-2} \mathrm{~s}^{-1}\right)$ provided by $40 \mathrm{~W}$ white fluorescent lamps. Root biomass growth and phenolic compound production were measured at 25 days of culture. The roots were separated from the media, and their fresh mass (FM) were determined after they were washed with water and the excess surface water blotted away, and dry mass (DM) were recorded after the roots were dried at $60{ }^{\circ} \mathrm{C}$ till constant weight is recorded.

\section{Analytical methods of phenolic compound in} hairy root cultures

Extraction and estimation of individual phenolic compounds by ultra-high-performance liquid chromatography (UHPLC)

Dried hairy roots powder $(1 \mathrm{~g})$ was extracted in acetonitrile $(10 \mathrm{~mL})$ and $0.1 \mathrm{~N}$ hydrochloric acid $(2 \mathrm{~mL})$. The extraction procedure was described ${ }^{16}$. The presence of twenty-four phenolic compounds in the hairy root cultures was measured using ultra-high-performance liquid chromatography (Accela Ultra-HPLC system, New York, USA) with a reverse phase column (Accela, $\mathrm{C}_{18}, 2.1 \times$ $100 \mathrm{~mm}, 2.6 \mathrm{~mm})$. The solvent, standard and gradient procedure was described ${ }^{18}$. Phenolic compounds in hairy root culture extracts were identified based on the retention time and UV spectra of the authentical standards, whereas the quantitative data were calculated based on the calibration curves of the individual standards. The results were expressed as $\mu \mathrm{g} / \mathrm{g}$ dry mass (DM) proportions of each compound that make up the total phenolic compound content.

\section{Estimation of total phenolic and flavonoid contents (TPC and TFC)}

Total phenolic content was quantified by a spectrophotometric method according to the Folin-Ciocalteu assay as previously reported ${ }^{19}$. The concentration of the TPC was calculated as mg of gallic acid equivalent by using an equation obtained from the gallic acid calibration curve. Total flavonoid content of the extracts was determined by using the aluminum chloride spectrophotometric method as described ${ }^{16}$. Quercetin used as the calibration curve and calculated as mg of quercetin equivalent.

\section{Biological activities in hairy root cultures Antioxidant activities}

The DPPH free-radical-scavenging activity, reducing power, phosphomolybdenum method, and metal ion-chelating assay were measured using a prior method ${ }^{18,20}$.

\section{Antibacterial and antifungal activities}

The pathogenic microorganisms Gram-positive bacteria (Staphylococcus aureus and Bacillus subtilis), Gram-negative bacteria (Pseudomonas aeruginosa and Escherichia coli) were used to test for antibacterial activity. Antibacterial tests were carried out by the NCCLS disc diffusion method was previously described ${ }^{5,18}$. The pathogenic fungi Candida albicans, Aspergillus niger, and Fusarium oxysporum were used to test for antifungal activity. Antifungal tests were carried out by the NCCLS disc diffusion method was previously reported $^{5,18}$. For antibacterial and antifungal activity was expressed as the relative inhibition percentage, and was calculated using the following equation:

Relative inhibition $(\%)=(\mathrm{X}-\mathrm{Y}) /(\mathrm{Z}-\mathrm{Y}) \times 100$

Where $X$ is the total area of inhibition of the test extract, $\mathrm{Y}$ is the total area of inhibition of the solvent and $\mathrm{Z}$ is the total area of inhibition of the standard drug.

\section{Cytotoxic screening of MTT assay}

Two human cancer cell lines, namely colon HT-29 (human colorectal adenocarcinoma) and oestrogen-dependent breast MCF-7 (human breast adenocarcinoma) cancer cells were used for cytotoxicity screening of the non-elicited and elicited hairy root extracts (JA and SA) in $M$. charantia. The cell lines cultured in DMEM medium supplemented with $10 \%$ FBS and incubated at $37^{\circ} \mathrm{C}$ in a humidified incubator with $5 \% \mathrm{CO}_{2}$. The cells were washed with PBS and then treated with trypsin. The culture was centrifuged, and the pellet was re-suspended in fresh medium. Briefly, cells $\left(5 \times 10^{3}\right.$ cells well $\left.^{-1}\right)$ were plated in 96 well plates and treated with different concentrations of non-elicited and elicited hairy root extracts $(12.5,25,50,100$ and $200 \mu \mathrm{g} / \mathrm{mL}$ ) for $48 \mathrm{~h}$. The control was used as solvent DMSO treated cells. Cell viability was determined using MTT [3-(4,5-dimethylthiazol-2- 
yl)-2,5-diphenyl tetrazolium bromide] colorimetric assay was performed to evaluate the cytotoxicity according to the method ${ }^{21}$ with slight modifications. The cells were treated with MTT reagent $(20 \mu \mathrm{L} /$ well $)$ for four $\mathrm{h}$ at $37^{\circ} \mathrm{C}$ and then DMSO $(200 \mu \mathrm{L})$ was added to dissolve the formazan crystals. The optical density (OD) was recorded at $492 \mathrm{~nm}$ in a microplate reader. The percentage inhibition of cell growth was calculated using the following formula: $100-\left(\mathrm{A}_{\text {sample }} / \mathrm{A}\right.$ control $\times 100$ ).

\section{Experimental design and data analysis}

All experiments were performed in triplicate, and each experiment was repeated three times and expressed as a mean \pm standard deviation (SD). One-way ANOVA analysis followed by Duncan's test was used to determine significant differences $(P \leq 0.05)$. All statistical analyses were performed using the SPSS Ver. 20 statistical software package.

\section{RESULTS AND DISCUSSION}

\section{Effects of JA and SA on biomass accumulation} in hairy root cultures

The biomass (fresh and dry mass) accumulation of JA and SA $(0,25,50$ and $100 \mu \mathrm{M})$ elicited and non-elicited hairy roots were studied in $M$. charantia (Table 1). The hairy roots biomass (91.15 FM and 10.05 DM) was decreased at higher concentrations $(100 \mu \mathrm{M})$ of JA when compared to non-elicited hairy roots (93.58 FM and 10.12 DM). Consistent with our results JA elicitation failed to increase the biomass of hairy roots compared to control roots of Sinapis alba ${ }^{22}$. The growth of hairy roots was suppressed at higher concentrations of JA in W. somnifera ${ }^{14,23}$. The biomass (95.75 FM and 10.20 DM) of hairy roots was increased at higher concentration $(100 \mu \mathrm{M})$ of SA when compared to non-elicited hairy roots (93.58 FM and 10.12 DM). Similar to our results SA-elicited hairy roots increased the biomass accumulation than non-elicited hairy roots of Withania somnifera ${ }^{14}$. SA at $100 \mu \mathrm{M}$ did not influence the biomass accumulation of hairy root cultures in Cichorium intybus ${ }^{24}$. Our results demonstrated that higher concentration of JA decreased whereas SA elicitation increased the biomass accumulation.
Table 1 - Effects of JA and SA on biomass accumulation in hairy root cultures of $M$. charantia.

\begin{tabular}{lll}
\hline Elicitation & \multicolumn{2}{c}{ Hairy roots } \\
SA & Fresh mass $(\mathrm{g} / \mathrm{L})$ & Dry mass $(\mathrm{g} / \mathrm{L})$ \\
\hline 0 & $93.58 \pm 1.00^{\mathrm{c}}$ & $10.12 \pm 0.1^{\mathrm{c}}$ \\
25 & $93.58 \pm 2.00^{\mathrm{c}}$ & $10.12 \pm 0.2^{\mathrm{c}}$ \\
50 & $95.62 \pm 1.50^{\mathrm{b}}$ & $10.15 \pm 0.1^{\mathrm{b}}$ \\
100 & $95.75 \pm 1.00^{\mathrm{a}}$ & $10.20 \pm 0.1^{\mathrm{a}}$ \\
$\mathrm{JA}$ & Fresh mass $(\mathrm{g} / \mathrm{L})^{\mathrm{D}}$ & Dry mass $(\mathrm{g} / \mathrm{L})$ \\
0 & $93.58 \pm 1.00^{\mathrm{a}}$ & $10.12 \pm 0.1^{\mathrm{a}}$ \\
25 & $93.58 \pm 2.00^{\mathrm{a}}$ & $10.12 \pm 0.2^{\mathrm{a}}$ \\
50 & $92.65 \pm 1.50^{\mathrm{b}}$ & $10.08 \pm 0.1^{\mathrm{b}}$ \\
100 & $91.15 \pm 1.00^{\mathrm{c}}$ & $10.05 \pm 0.1^{\mathrm{c}}$ \\
\hline
\end{tabular}

Means \pm standard deviations (SD) of three replicates followed by the same letters are not significantly different according to Duncan's multiple range test at $P$ $\leq 0.05$.

\section{Effects of JA and SA on phenolic compound profiles in hairy root cultures}

Hairy root cultures are a promising system for the production of valuable secondary metabolites such as pharmaceuticals, pigments, and flavors in many plants $^{25}$. SA and JA are messengers in the signal transduction chains involved in different pathways of biosynthesis and inactivation of secondary metabolites and enzymes that participate in the plant stress response, and exogenous application of JA and SA may also induce the expression of many defense genes in plants ${ }^{18}$. JA and SA as important signal molecules in systemic acquired resistance have been used to enhance the production of secondary metabolites in hairy root cultures. JA and SA stimulated the phenylpropanoid pathway genes that lead to the accumulation of phenolic compounds ${ }^{26}$. In this study revealed the phenolic compound production using non-elicited and elicited (JA and SA) hairy root cultures of $M$. charantia. Previously, it was reported the enhanced production of phytochemicals in methyl jasmonate (MeJA) elicited-hairy root cultures of Salvia miltiorrhiza ${ }^{11}$. Elicitation JA at $100 \mathrm{mM}$ resulted in a six to ninefold increase in the production of azadirachtin in hairy root cultures of Azadirachta indica ${ }^{12}$. We studied the effect of elicitors (JA and SA) in individual phenolic compounds, total phenolic and flavonoids in hairy root cultures of $M$. charantia.

\section{Individual phenolic compounds}

The qualitative and quantitative analyses of phenolic compounds from non-elicited and elicited (JA and SA) hairy root culture extracts were performed using UHPLC (Table 2). The phenolic 
compounds in the hairy root extracts were identified by comparison of their retention time and UV spectra with that of authentic standards, and the quantitative data were calculated from calibration curves. Both non-elicited and elicited (JA and SA) hairy roots contained flavonols, hydroxybenzoic and hydroxycinnamic acids (Table 2). JA and SA-elicited hairy roots contained a higher level of hydroxybenzoic acid $(8444.83$ and $7960.02 \mu \mathrm{g} / \mathrm{g}$ ) than non-elicited hairy roots $(7411.63 \mu \mathrm{g} / \mathrm{g})$. The contents of gentisic, salicylic, gallic, $p$-hydroxybenzoic, protocatechuic, vanillic, syringic and $\beta$-resorcylic acids were significantly higher in JA and SA-elicited hairy root cultures than that in non-elicited hairy root cultures $(P \leq 0.05$; Table 2$)$. JA and SA were enhanced the phenolic acids composition in Vitis vinifera suspension culture ${ }^{27}$. JA and SA-elicited hairy roots produced a higher amount of hydroxycinnamic acid (1224.26 and $1090.63 \mu \mathrm{g} / \mathrm{g}$ ) when compared to non-elicited hairy roots (997.04 $\mu \mathrm{g} / \mathrm{g}$ ). The concentration of chlorogenic, ferulic, caffeic, $p$-coumaric and $t$-cinnamic acids were significantly increased in JA and SA-elicited hairy roots compared to non-elicited hairy roots $(P \leq$ 0.05 ; Table 2). Consistently, SA-elicited hairy root cultures enhanced rosmarinic acid production about 1.87 -fold compared to the non-elicited hairy roots of Salvia miltiorrhiza ${ }^{28}$, and $100 \mu \mathrm{M}$ SA enhanced rosmarinic acid production about twofold in hairy root cultures of Coleus forskohlii ${ }^{29}$. SA has also been demonstrated positively to influence the biosynthetic pathway of phenolic $\operatorname{acids}^{28}$. Similar to our results, caffeic acid content was higher in SA-elicited cell cultures of $S$. miltiorrhiz $a^{30}$. JA and SA-elicited hairy roots formed the higher amount of flavonols (2680.88 and $2489.57 \mu \mathrm{g} / \mathrm{g}$ ) than non-elicited hairy roots $(2190.90 \mu \mathrm{g} / \mathrm{g})$. The amount of catechin, rutin, myricetin, naringenin, quercetin, kaempferol and biochanin A were significantly higher in JA and SA-elicited hairy roots than that in non-elicited hairy roots $(P \leq 0.05$; Table 2$)$. MeJA increased accumulation of isoflavones to about five times higher than that for control shoots of Pueraria montana $^{32}$. Daidzin content enhancement up to 7.3-fold was obtained with $10 \mu \mathrm{M} \mathrm{JA}$ treatment in hairy root cultures of Psoralea corylifolia compared in control $^{33}$. Pueraria candollei hairy root culture treatment with SA at $100 \mu \mathrm{M}$ resulted in higher yield of isoflavonoid compared to control after three days of elicitation ${ }^{34}$. Veratric acid, vanillin, hesperidin and homogentisic acid were significantly increased in JA and SA-elicited hairy root cultures compared to non-elicited hairy root cultures $(P \leq 0.05$; Table 2$)$.

Table 2 - Ultra-HPLC analysis of the main phenolic compounds from elicited (JA and SA) and non-elicited hairy root cultures of Momordica charantia.

\begin{tabular}{|c|c|c|c|c|}
\hline \multirow[t]{2}{*}{ No } & \multirow[t]{2}{*}{ Phenolic compounds } & \multicolumn{3}{|c|}{ Concentration $(\mu \mathrm{g} / \mathrm{g} \mathrm{DM})$} \\
\hline & & $\begin{array}{c}\text { Non-elicited hairy } \\
\text { roots }\end{array}$ & $\begin{array}{c}\text { Elicited hairy roots } \\
(\text { SA } 100 \mu \mathrm{M})\end{array}$ & $\begin{array}{c}\text { Elicited hairy roots } \\
(\mathrm{JA} 100 \mu \mathrm{M})\end{array}$ \\
\hline & Hydroxybenzoic acid & & & \\
\hline 1 & $p$-Hydroxybenzoic acid & $141.41 \pm 1.2^{\mathrm{i}, \mathrm{z}}$ & $155.25 \pm 1.5^{\mathrm{k}, \mathrm{y}}$ & $179.15 \pm 1.0^{\mathrm{j}, \mathrm{x}}$ \\
\hline 2 & Gallic acid & $169.25 \pm 2.0^{\mathrm{h}, \mathrm{z}}$ & $194.12 \pm 2.0^{\mathrm{h}, \mathrm{y}}$ & $225.21 \pm 2.0^{\mathrm{h}, \mathrm{x}}$ \\
\hline 3 & Protocatechuic acid & $38.41 \pm 1.1^{\mathrm{q}, \mathrm{z}}$ & $56.10 \pm 1.0^{\mathrm{p}, \mathrm{y}}$ & $71.00 \pm 1.0^{\mathrm{p}, \mathrm{x}}$ \\
\hline 4 & Syringic acid & $75.16 \pm 1.2^{\mathrm{n}, \mathrm{z}}$ & $88.25 \pm 1.0^{\mathrm{n}, \mathrm{y}}$ & $96.52 \pm 1.5^{\mathrm{n}, \mathrm{x}}$ \\
\hline 5 & Gentisic acid & $4,553.65 \pm 2.5^{\mathrm{a}, \mathrm{z}}$ & $4,695.10 \pm 3.0^{\mathrm{a}, \mathrm{y}}$ & $4,850.00 \pm 2.0^{\mathrm{a}, \mathrm{x}}$ \\
\hline 6 & Salicylic acid & $2,241.00 \pm 2.1^{\mathrm{b}, \mathrm{z}}$ & $2,550 \cdot 20 \pm 2.0^{\mathrm{b}, \mathrm{y}}$ & $2,775.50 \pm 2.0^{\mathrm{b}, \mathrm{x}}$ \\
\hline 7 & Vanillic acid & $130.00 \pm 2.5^{\mathrm{j}, \mathrm{z}}$ & $145.50 \pm 1.0^{1, y}$ & $159.45 \pm 1.0^{1, \mathrm{x}}$ \\
\hline \multirow[t]{3}{*}{8} & $\beta$-Resorcylic acid & $62.75 \pm 1.1^{\mathrm{o}, \mathrm{z}}$ & $75.50 \pm 1.0^{\mathrm{o}, \mathrm{y}}$ & $88.00 \pm 1.0^{\mathrm{o}, \mathrm{x}}$ \\
\hline & Total & $7411.63^{\mathrm{z}}$ & $7960.02^{y}$ & $8444.83^{\mathrm{x}}$ \\
\hline & Hydroxycinnamic acid & & & \\
\hline 9 & Caffeic acid & $143.50 \pm 1.3^{\mathrm{i}, \mathrm{z}}$ & $165.00 \pm 2.0^{\mathrm{j}, \mathrm{y}}$ & $234.25 \pm 2.0^{\mathrm{h}, \mathrm{x}}$ \\
\hline 10 & $p$-Coumaric acid & $125.12 \pm 1.2^{\mathrm{k}, \mathrm{z}}$ & $141.23 \pm 1.0^{1, \mathrm{y}}$ & $159.50 \pm 1.0^{1, \mathrm{x}}$ \\
\hline 11 & Ferulic acid & $194.05 \pm 1.0^{\mathrm{g}, \mathrm{z}}$ & $217.15 \pm 1.5^{\mathrm{g}, \mathrm{y}}$ & $228.20 \pm 1.5^{\mathrm{h}, \mathrm{x}}$ \\
\hline 12 & Chlorogenic acid & $531.12 \pm 1.0^{\mathrm{d}, \mathrm{z}}$ & $555.15 \pm 1.0^{\mathrm{d}, \mathrm{y}}$ & $584.31 \pm 1.0^{\mathrm{d}, \mathrm{x}}$ \\
\hline \multirow[t]{3}{*}{13} & $t$ - Cinnamic acid & $3.25 \pm 0.21^{\mathrm{s}, \mathrm{z}}$ & $12.10 \pm 0.5^{\mathrm{s}, \mathrm{y}}$ & $18.00 \pm 0.8^{\mathrm{s}, \mathrm{x}}$ \\
\hline & Total & $997.04^{\mathrm{z}}$ & $1090.63^{\mathrm{y}}$ & $1224.26^{\mathrm{x}}$ \\
\hline & Flavonols & & & \\
\hline
\end{tabular}




\begin{tabular}{|c|c|c|c|c|}
\hline 14 & Myricetin & $224.15 \pm 3.1^{\mathrm{f}, \mathrm{z}}$ & $262.30 \pm 2.0^{\mathrm{f}, \mathrm{y}}$ & $315.21 \pm 1.5^{\mathrm{f}, \mathrm{x}}$ \\
\hline 15 & Quercetin & $99.12 \pm 1.0^{\mathrm{l}, \mathrm{z}}$ & $171.12 \pm 2.0^{\mathrm{i}, \mathrm{y}}$ & $215.50 \pm 2.0^{\mathrm{i}, \mathrm{x}}$ \\
\hline 16 & Kaempferol & $88.25 \pm 0.5^{\mathrm{m}, \mathrm{z}}$ & $141.00 \pm 1.5^{1, y}$ & $169.00 \pm 2.0^{\mathrm{k}, \mathrm{x}}$ \\
\hline 17 & Catechin & $1,321.12 \pm 4.0^{\mathrm{c}, \mathrm{z}}$ & $1,350.00 \pm 2.0^{\mathrm{c}, \mathrm{y}}$ & $1,375.55 \pm 2.0^{\mathrm{c}, \mathrm{x}}$ \\
\hline 18 & Rutin & $315.47 \pm 1.4^{\mathrm{e}, \mathrm{z}}$ & $402.00 \pm 2.0^{\mathrm{e}, \mathrm{y}}$ & $425.50 \pm 2.0^{\mathrm{e}, \mathrm{x}}$ \\
\hline 19 & Naringenin & $121.32 \pm 1.2^{\mathrm{k}, \mathrm{z}}$ & $134.00 \pm 1.0^{\mathrm{m}, \mathrm{y}}$ & $145.12 \pm 1.0^{\mathrm{m}, \mathrm{x}}$ \\
\hline \multirow[t]{3}{*}{20} & Biochanin A & $21.47 \pm 0.9^{\mathrm{r}, \mathrm{z}}$ & $29.15 \pm 1.0^{\mathrm{r}, \mathrm{y}}$ & $35.00 \pm 1.0^{\mathrm{r}, \mathrm{x}}$ \\
\hline & Total & $2190.90^{z}$ & $2489.57^{y}$ & $2680.88^{x}$ \\
\hline & Other phenolic constit & & & \\
\hline 21 & Vanillin & $67.21 \pm 1.2^{\mathrm{o}, \mathrm{z}}$ & $75.00 \pm 1.0^{\mathrm{o}, \mathrm{y}}$ & $90.15 \pm 1.0^{\mathrm{o}, \mathrm{x}}$ \\
\hline 22 & Veratric acid & $215.14 \pm 1.0^{\mathrm{f}, \mathrm{z}}$ & $225.00 \pm 1.0^{\mathrm{g}, \mathrm{y}}$ & $255.11 \pm 1.0^{\mathrm{g}, \mathrm{x}}$ \\
\hline 23 & Homogentisic acid & $36.75 \pm 1.1^{\mathrm{q}, \mathrm{z}}$ & $45.15 \pm 0.5^{\mathrm{q}, \mathrm{y}}$ & $52.00 \pm 0.5^{\mathrm{q}, \mathrm{x}}$ \\
\hline \multirow[t]{2}{*}{24} & Hesperidin & $45.58 \pm 1.0^{\mathrm{p}, \mathrm{z}}$ & $55.00 \pm 1.0^{\mathrm{p}, \mathrm{y}}$ & $64.00 \pm 0.7^{\mathrm{p}, \mathrm{x}}$ \\
\hline & Total & $364.68^{z}$ & $399.15^{\mathrm{y}}$ & $461.26^{\mathrm{x}}$ \\
\hline
\end{tabular}

Mean \pm standard deviations (SD) of three replicates within a column ${ }^{\mathrm{a}-\mathrm{s}}$, or row $^{\mathrm{x}-\mathrm{z}}$ followed by the same letters are not significantly different according to Duncan's multiple range test at $P \leq 0.05$.

Total phenolic (TPC) and flavonoid contents (TFC)

The present study reported that JA and SA-elicited hairy roots could prominently induce the accumulation of the total phenolics and flavonoids. Figure 1A exhibits the contents of TPC were significantly increased the amount of $4.1 \mathrm{mg} / \mathrm{g}$ and $3.7 \mathrm{mg} / \mathrm{g}$ gallic acid equivalent (GAE) in JA and SA-elicited hairy root cultures than non-elicited hairy root cultures $(2.8 \mathrm{mg} / \mathrm{g}$ GAE). TFC of 3.5 $\mathrm{mg} / \mathrm{g}$ and $3.2 \mathrm{mg} / \mathrm{g}$ quercetin equivalent (QE) in $\mathrm{JA}$ and SA-elicited hairy root cultures were higher compared to non-elicited hairy root cultures (2.5 $\mathrm{mg} / \mathrm{g}$ QE; Figure 1B). Elicitation of MeJA was increased the amount TPC and TFC in hairy roots than non-elicited hairy root cultures of Ginseng and Echinacea, respectively ${ }^{35}$. JA and SA elicitorsinduced enhancement in phenolic and flavonoid accumulation in suspension cultures of Artemisia absinthium $^{36}$. Although the phenolic compounds concentration was increased under SA elicitation, maximum production of phenolic compounds was obtained in JA-elicited hairy root cultures. Likewise, MeJA was more effective than SA in soybean cell suspension culture ${ }^{37}$. Our results suggest that JA and SA elicitation was increased the level of individual and total phenolic compounds in hairy root cultures of $M$. charantia.

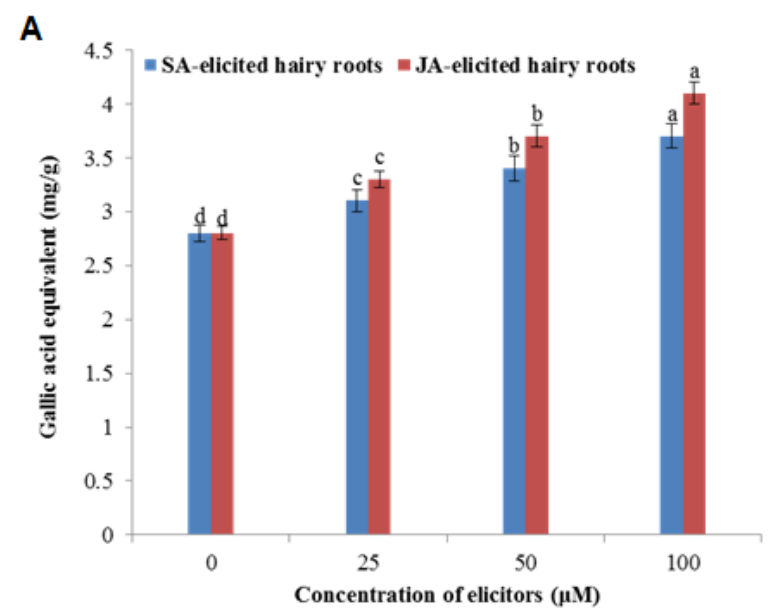

B

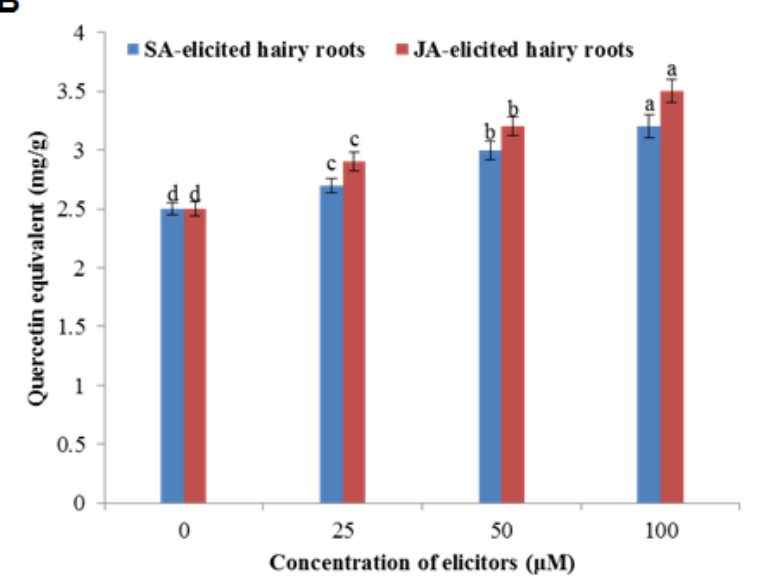

Figure 1 - Effects of JA and SA on levels of total phenolic and flavonoid contents (TPC and TFC) in hairy root cultures of $M$. charantia. A) TPC, B) TFC. Means \pm SD of three replicates followed by the same letters are not significantly different according to Duncan's multiple range test at $P \leq 0.05$. 
Phenolic compounds of hairy roots in bitter melon

Effects of JA and SA on antioxidant activity of hairy root cultures

Antioxidant potential of medicinal and food plant extracts has been qualified to the presence of phenolic compounds ${ }^{16}$. Phenolic compounds were playing an important role in determining antioxidant capacity of various plant species. Antioxidant activity is mainly due to phenolic compounds redox properties, which play a major role in adsorbing and neutralizing free radicals, quenching singlet and triplet oxygen or decomposing peroxides ${ }^{38}$. The biosynthetic enzymes antioxidants were found upregulated by the application of $\mathrm{SA}^{31}$. Due to the presence of phenolic compounds were the primarily antioxidant contributors of $M$. charantia, which can act as the hydrogen/electron donors to neutralize peroxyl free radicals. The antioxidant capacity of JA and SA-elicited hairy root cultures were evaluated using free radicals scavenging, reducing potential, phosphomolybdenum assay and metal chelating activity. Figure $2 \mathrm{~A}$ shows the high antioxidant activity was exhibited in JA $(84.00 \%)$ and SA $(78.00 \%)$ elicited hairy roots compared with non-elicited hairy roots $(69.00 \%)$. The phenolic and flavonoid level was higher in the JA, and SA-elicited hairy root cultures, which directly influence their antioxidant potential. Figure 2B displays the reducing capacity of $M$. charantia hairy root culture extracts suggested that JA and SA-elicited cultures had the more antioxidant potential than non-elicited hairy roots. The antioxidant capacity of elicited (JA and SA) and non-elicited hairy root extracts was determined using the phosphomolybdenum method. The antioxidant capability of the elicited (JA and SA) hairy roots was higher when compared to non-elicited hairy root extracts (Fig. 2C). The chelating agent may inhibit radical generation by stabilizing transition metals, thus reducing free radical damage. Ferrozine can make complexes with ferrous ions. In the existence of a chelating agent complex (red color) formation is interrupted and as a result, the red color of the compound decreases. Figure 2D shows the percentage of metal scavenging capacity exhibited in JA $(80.00 \%)$ and SA $(74.00 \%)$ was high compared with that of non-elicited hairy root extracts $(62.00 \%)$. Consistent with our reports, JA and SA-elicited suspension cells increased the antioxidant activity in $A$. absinthium ${ }^{36}$.

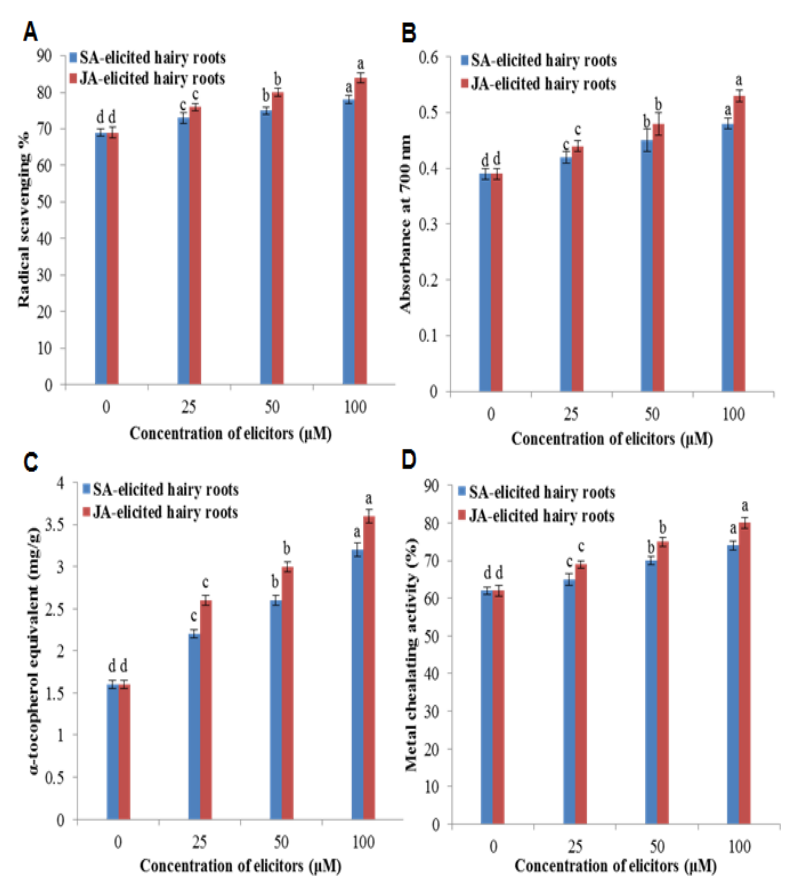

Figure 2 - Effects of JA and SA on levels of antioxidant activity in hairy root cultures of $M$. charantia. A) Free radical-scavenging activity by DPPH method, B) Reducing power, C) Phosphomolybdenum method, D) Metal chelating activity. Means \pm SD of three replicates followed by the same letters are not significantly different according to Duncan's multiple range test at $P \leq 0.05$.

\section{Effects of JA and SA on antimicrobial activity of hairy root cultures}

Elicited (JA and SA) non-elicited hairy root cultures presented variable antimicrobial activity as revealed by the growth inhibition zones (Fig. 3A). The disc diffusion method results indicated that elicited (JA and SA) and non-elicited hairy root culture extracts had anti-bacterial (Grampositive and Gram-negative bacteria) and antifungal activities. Figure 3A shows the elicited (JA and SA) hairy root culture extracts exhibited higher activity against antibacterial and antifungal compared to control hairy root extracts of $M$. charantia. Similarly, JA and SA-elicited cell suspension cultures increased antibacterial and antifungal activity ${ }^{39}$. These results were compared with chloramphenicol and thymol for positive control activity of bacteria and fungus respectively. Our study showed that JA and SA elicitation was increased the level of biological activities (antioxidant and antimicrobial) in hairy root cultures of $M$. charantia. The results proved that hairy root culture extracts of $M$. charantia 
could be used for the treatment of bacterial and fungal diseases.

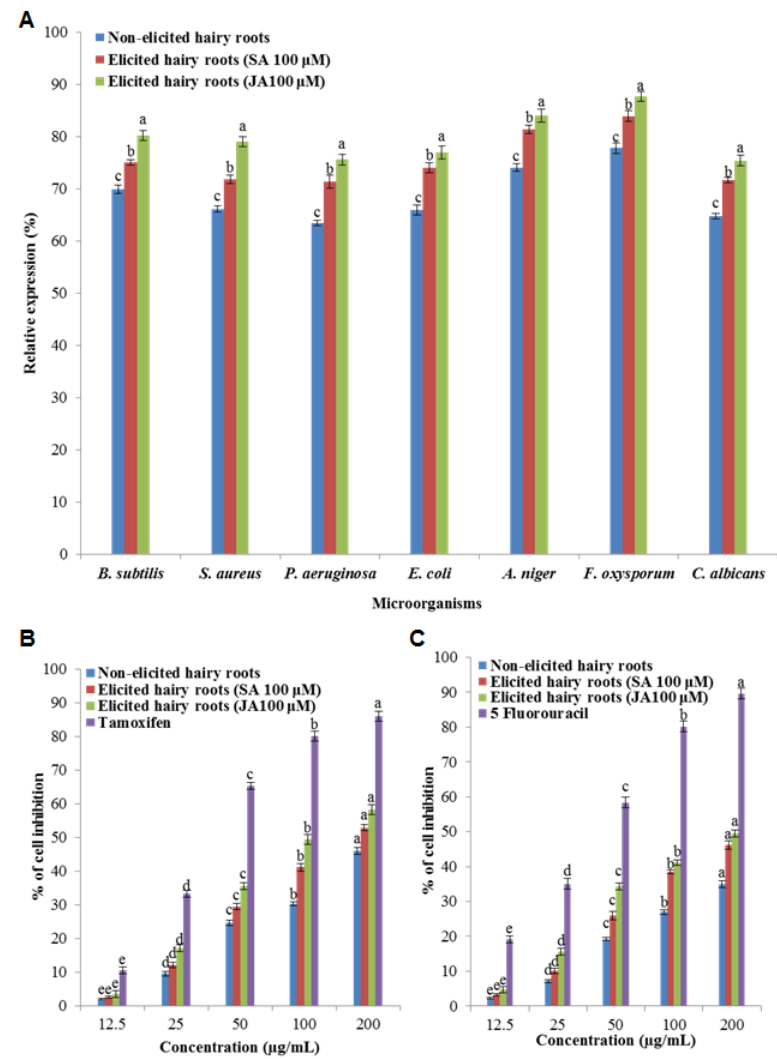

Figure 3 - Effects of JA and SA on levels of antimicrobial and anticancer activity in hairy root cultures of $M$. charantia. A) Antimicrobial activity using disc diffusion method, B) MCF-7 cell line, C) HT-29 cell line. Means \pm SD of three replicates followed by the same letters are not significantly different according to Duncan's multiple range test at $P$ $\leq 0.05$.

\section{Effects of JA and SA on anticancer activity of hairy root cultures}

There are many studies reported on the phenolic compound of rosmarinic acid activity against Herpes simplex virus 1 (HSV-1), Human immunodeficiency virus (HIV-1) and Helicobacter pylori $^{38}$. Anticancer activities against MCF-7 and HT-29 cell lines in elicited (JA and SA) and nonelicited hairy roots were evaluated. The inhibition of these extracts are compared with standard tamoxifen for MCF-7 cell line and 5-fluorouracil for HT-29 cell line (Fig. 3B, C) respectively. The percentage of cancer cell inhibition profiles were found to be concentration dependent, and the greater inhibition was a higher concentration of $200 \mu \mathrm{g} / \mathrm{mL}$. MCF-7 cell lines grown in DMEM, when exposed to different concentrations of JA and SA-elicited hairy roots extracts resulted in
$58.25 \%$ and $52.98 \%$ inhibition of MCF-7 cell death whereas non-elicited hairy roots displayed weak inhibition of $46.02 \%$. On the other hand, comparison with tamoxifen showed that $88.16 \%$ MCF-7 cell line inhibition at the same tested dose $(200 \mu \mathrm{g} / \mathrm{mL})$. JA and SA-elicited hairy roots and non-elicited hairy root extracts tested against HT29 cell line resulted in anticancer activity profile than the standard 5-fluorouracil. A maximum concentration of $49.50 \%$ and $46.09 \%$ inhibition was observed with JA and SA-elicited hairy root extracts at a tested dose of $200 \mu \mathrm{g} / \mathrm{mL}$. MCF-7 cell line was greater inhibition than HT-29 cell line (Fig. 3B, C). Similarly, hairy roots exhibited higher anticancer activity compared to nontransformed roots in Dracocephalum moldavica ${ }^{38}$ and Lithospermum canescens ${ }^{40}$. In this study shows the MTT assay resulted that hairy root cultures of bitter melon can inhibit the growth of breast and colon cancer cell lines. This inhibition of cell growth could be attributed to its phenolic compounds content. Our results suggest that transformed hairy roots can be effectively used for the antioxidant, antimicrobial and anticancer treatments.

\section{CONCLUSION}

JA and SA-elicited hairy roots produced a higher amount of individual phenolic compounds, total phenolic, and flavonoids when compared to nonelicited hairy roots. The antioxidant, antimicrobial and anticancer activities were higher in JA and SA-elicited hairy roots than that in non-elicited hairy roots. The elevated level of phenolic compounds probably contributes to the marked antioxidant, antibacterial, antifungal and anticancer activity of hairy roots in bitter melon. These results demonstrate that the application of elicitors allows the optimized production of phenolic compounds in hairy root cultures of $M$. charantia. To the best of our knowledge, this is the first report describing the phenolic compound production and biological activities from JA and SA-elicited hairy root cultures of $M$. charantia. This protocol will be useful for biochemical and bioprocess engineering for the sustainable production of valuable phytochemicals in hairy root culture. 
Phenolic compounds of hairy roots in bitter melon

\section{ACKNOWLEDGMENT}

This paper was supported by the KU Research Professor Program of Konkuk University, Seoul, South Korea.

\section{REFERENCES}

1-Grover JK, Yadav SP. Pharmacological actions and potential uses of Momordica charantia: A review. $J$ Ethnopharmacol. 2004; 93: 123-132.

2-Tan SP, Kha TC, Parks SE, Roach PD. Bitter melon (Momordica charantia L.) bioactive composition and health benefits: A review. Food Reviews International. 2016; 32(2): 181-202.

3-Ignat I, Volf I, Popa VI. A critical review of methods for characterization of polyphenolic compounds in fruits and vegetables. Food Chem. 2011; 126: 18211835.

4-Kenny O, Smyth TJ, Hewage CM, Brunton NP. Antioxidant properties and quantitative UPLC-MS analysis of phenolic compounds from extracts of fenugreek (Trigonella foenum-graecum) seeds and bitter melon (Momordica charantia) fruit. Food Chem. 2013; 141(4): 4295-302.

5-Yoon JY, Chung IM, Thiruvengadam M. Evaluation of phenolic compounds, antioxidant and antimicrobial activities from transgenic hairy root cultures of gherkin (Cucumis anguria L.). South Afr J Bot. 2015; 100: 80-86.

6-Thwe A, Arasu MV, Li X, Park CH, Kim SJ, AlDhabi NA, Park SU. Effect of different Agrobacterium rhizogenes strains on hairy root induction and phenylpropanoid biosynthesis in tartary buckwheat (Fagopyrum tataricum Gaertn). Front Microbiol. 2016; 7: 318.

7-Thiruvengadam M, Rekha K, Chung IM. Induction of hairy roots by Agrobacterium rhizogenesmediated transformation of spine gourd (Momordica dioica Roxb. ex. willd) for the assessment of phenolic compounds and biological activities. Sci. Hortic. 2016; 198: 132-141.

8-Giri CC, Zaheer M. Chemical elicitors versus secondary metabolite production in vitro using plant cell, tissue and organ cultures: recent trends and a sky eye view appraisal. Plant Cell Tiss Org Cult. 2016; 1-18.

9-Ooi CT, Syahida A, Stanslas J, Maziah M. The influence of methyl jasmonate, cholesterol and 1arginine on solasodine production in hairy root culture of Solanum mammosum. Eng Life Sci. 2016; 00: 1-11.

10- Li B, Wang B, Li H, Peng L, Ru M, Liang Z, Yan X, Zhu Y. Establishment of Salvia castanea Diels f. tomentosa Stib. hairy root cultures and the promotion of tanshinone accumulation and gene expression with $\mathrm{Ag}+$, methyl jasmonate, and yeast extract elicitation. Protoplasma 2016; 253(1): 87100 .

11- Kai G, Liao P, Xu H, Wang J, Zhou C, Zhou W, Qi Y, Xiao J, Wang Y, Zhang L. Molecular mechanism of elicitor-induced tanshinone accumulation in Salvia miltiorrhiza hairy root cultures. Acta Physiol Plant. 2012; 34: 1421-1433.

12- Satdive RK, Fulzele DP, Eapen S. Enhanced production of azadirachtin by hairy root cultures of Azadirachta indica A. Juss by elicitation and media optimization. J Biotechnol. 2007; 128: 281-289.

13- Spollansky TC, Pitta-Alvarez SI, Giulietti AM. Effect of jasmonic acid and aluminium on production of tropane alkaloids in hairy root cultures of Brugmansia candida. Electronic J Biotechnol. 2000; 3(1): 72-75

14- Sivanandhan G, Kapil Dev G, Jeyaraj M, Rajesh M, Muthuselvam M, Selvaraj N, Manickavasagam M, Ganapathi A. A promising approach on biomass accumulation and withanolides production in cell suspension culture of Withania somnifera (L.) Dunal. Protoplasma 2013; 250: 885898.

15- Nair VD, Panneerselvam R, Gopi R, Shao HB. Elicitation of pharmacologically active phenolic compounds from Rauvolfia serpentina Benth. Ex. Kurtz. Ind Crop Prod. 2013; 45: 406-415.

16- Thiruvengadam M, Praveen N, Maria John KM, Yang YS, Kim SH, Chung IM. Establishment of Momordica charantia hairy root cultures for the production of phenolic compounds and determination of their biological activities. Plant Cell Tiss Org Cult. 2014; 118: 545-557.

17- Murashige T, Skoog F. A revised medium for rapid growth and bioassays with tobacco tissue cultures. Physiol Plant. 1962; 15: 473-497.

18- Thiruvengadam M, Kim SH, Chung IM. Exogenous phytohormones increase the accumulation of health-promoting metabolites, and influence the expression patterns of biosynthesis related genes and biological activity in Chinese cabbage (Brassica rapa spp. pekinensis). Sci Hortic. 2015; 193: 136-146.

19- Ceylan O, Alıc H. Antibiofilm, antioxidant, antimutagenic activities and phenolic compounds of Allium orientale BOISS. Braz Arch Biol Technol. 2015; 58(6): 935-943.

20- Pal RS, Kumar VA, Arora S, Sharma AK, Kumar V, Agrawal S. Physicochemical and antioxidant properties of kiwifruit as a function of cultivar and fruit harvested month. Braz Arch Biol Technol. 2015; 58 (2): 262-271.

21- Gabr AMM, Mabrok HB, Ghanem KZ, Blaut M, Smetanska I. Lignan accumulation in callus and Agrobacterium rhizogenes mediated hairy root cultures of flax (Linum usitatissimum). Plant Cell Tiss Org Cult. 2016; 126: 255-267. 
22- Kastell A, Smetanska I, Ulrichs C, Cai Z, Mewis I. Effects of phytohormones and jasmonic acid on glucosinolate content in hairy root cultures of Sinapis alba and Brassica rapa. Appl Biochem Biotechnol. 2013; 169: 624-635.

23- Doma M, Abhayankar G, Reddy VD, Kavi Kishor PB. Carbohydrate and elicitor enhanced withanolide (withaferin A and withanolide A) accumulation in hairy root cultures of Withania somnifera (L.). Indian J Exp Biol. 2012; 50: 484490.

24- Malarz J, Stojakowska A, Kisiel W Effect of methyl jasmonate and salicylic acid on sesquiterpene lactone accumulation in hairy roots of Cichorium intybus. Acta Physiol Plant. 2007; 29: 127-132.

25- Srivastava S, Srivastava AK. Hairy root culture for mass-production of high-value secondary metabolites. Crit Rev Biotechnol. 2007; 27: 29-43.

26- Thiruvengadam M, Baskar V, Kim SH, Chung IM. Effects of abscisic acid, jasmonic acid and salicylic acid on the content of phytochemicals and their gene expression profiles and biological activity in turnip (Brassica rapa ssp. rapa). Plant Growth Regul. 2016; Doi: 10.1007/s10725-016-0178-7.

27- Riedel H, Akumo DN, Thaw Saw NMM, Kütük O, Neubauer P, Smetanska I. Elicitation and precursor feeding influence phenolic acids composition in Vitis vinifera suspension culture. Afr J Biotechnol. 2012; 11: 3000-3008.

28- Zhang S, Li H, Liang X, Yan Y, Xia P, Jia Y, Liang Z. Enhanced production of phenolic acids in Salvia miltiorrhiza hairy root cultures by combing the RNAi-mediated silencing of chalcone synthase gene with salicylic acid treatment. Biochem Eng J. 2015; 103: 185-192.

29- Li W, Koike K, Asada Y, Yoshikawa T, Nikaido T. Rosmarinic acid production by Coleus forskohlii hairy root cultures. Plant Cell Tiss Org Cult. 2005; 80: 151-155.

30- Cortell JM, Halbleib M, Gallagher AV, Righetti TL, Kennedy JA. Influence of vine vigor on grape (Vitis vinifera L. Cv. Pinot Noir) anthocyanins. 1. Anthocyanin concentration and composition in fruit. J Agric Food Chem. 2007; 55(16): 6575-6584.

31- Dong J, Wan G, Liang Z. Accumulation of salicylic acid-induced phenolic compounds and raised activities of secondary metabolic and antioxidative enzymes in Salvia miltiorrhiza cell culture. J Biotechnol. 2010; 148: 99-104.

32- Kirakosyan A, Kaufman P, Chang S, Warber $\mathrm{S}$, Bolling $\mathrm{S}$, Vardapetyan $\mathrm{H}$ Regulation of isoflavone production in hydroponically grown Pueraria montana (kudzu) by cork pieces, XAD-4, and methyl jasmonate. Plant Cell Rep. 2006; 25: 1387-1391.

33- Zaheer M, Reddy VD, Giri CC. Enhanced daidzin production from jasmonic and acetyl salicylic acid elicited hairy root cultures of Psoralea corylifolia L. (Fabaceae). Nat Prod Res. 2016; Doi:10.1080/14786419.2015.1054823.

34- Udomsuk L, Jarukamjorn $\mathrm{K}$, Tanaka $\mathrm{H}$, Putalun W. Improved isoflavonoid production in Pueraria candollei hairy root cultures using elicitation. Biotechnol Lett. 2011; 33: 369-374.

35- Wu CH, Murthy HN, Hahn EJ, Paek KY. Establishment of adventitious root co-culture of Ginseng and Echinacea for the production of secondary metabolites. Acta Physiol Plant. 2008; 30: 891-896.

36- Ali M, Abbasi BH, Ali GS. Elicitation of antioxidant secondary metabolites with jasmonates and gibberellic acid in cell suspension cultures of Artemisia absinthium L. Plant Cell Tiss Org Cult. 2015; 120: 1099-1106.

37- Theboral J, Sivanandhan G, Subramanyam K, Arun M, Selvaraj N, Manickavasagam M, Ganapathi A. Enhanced production of isoflavones by elicitation in hairy root culture of soybean. Plant Cell Tiss Org Cult. 2014; 117: 477-481.

38- Weremczuk-Jezyna I, Grzegorczyk-Karolak I, Frydrych B, Kro'licka A, Wysokin'ska H. Hairy roots of Dracocephalum moldavica: rosmarinic acid content and antioxidant potential. Acta Physiol Plant. 2013; 35: 2095-2103.

39- Abdelmohsen UR, Ali W, Eom SH, Hentschel U, Roitsch T. Synthesis of distinctly different sets of antimicrobial activities by elicited plant cell suspension cultures. Plant Cell Tiss Org Cult. 2010; 106: 105-113.

40- Syklowska-Baranek K, Pietrosiuk A, Gawron A, Kawiak A, Loikowska E, Jeziorek M, Chinou I. Enhanced production of antitumour naphthoquinones in transgenic hairy root lines of Lithospermum canescens. Plant Cell Tiss Org Cult. 2012; 108: 213219. 